\title{
Perfil de preferencias de aprendizaje de alumnos y profesores de medicina. Elemento a considerar en la enseñanza
}

\author{
M.E. Ponce de León-Castañeda, M. Varela-Ruiz, J.R. Lozano-Sánchez, A. Ortiz-Montalvo, R.E. Ponce-Rosas
}

Introducción. Es importante que alumnos y profesores comprendan y desarrollen habilidades que favorezcan un mejor aprendizaje. Para ello se debe identificar sus perfiles de aprendizaje. Sujetos y métodos. Con autorización del autor se aplicó el cuestionario VARK a 530 alumnos y 27 profesores del primer año de la carrera de Medicina en la Universidad Nacional Autónoma de México. Las respuestas al cuestionario se procesaron con la metodología establecida por el autor. Se utilizó la prueba exacta de Fisher para identificar diferencias entre ambos perfiles. Mediante análisis inferencial se buscó asociación por género y con el promedio de calificaciones, así como entre profesor y alumnos. Resultados. El 31\% de alumnos presentó una modalidad, y el 69\%, más de una; de éstos, el 14\% desarrolló las cuatro modalidades. El 41\% fueron visual/cinestésicos, y el $64 \%$ desarrolló una o dos modalidades. De los profesores, el 26\% manifestó una modalidad, y el $74 \%$, más de una; de ellos, el $4 \%$ tuvo las cuatro modalidades. No se encontraron diferencias estadísticamente significativas entre mujeres y hombres, ni entre los grupos matutinos, vespertinos o de alto rendimiento. Conclusiones. Este primer acercamiento permitió tener una visión general de las preferencias de aprendizaje de la población estudiada. Los resultados permitirán que cuando profesores y alumnos reconozcan su estilo de aprendizaje, podrán enriquecer su práctica docente y acercarla a las necesidades de sus alumnos, y con ello mejorar el proceso de enseñanza y los resultados del aprendizaje.

Palabras clave. Alumno. Estilos de aprendizaje. Profesor. VARK.

\section{Profile of learning preferences of students and professors of medicine. Element to consider in teaching}

Introduction. It is important for students and teachers to identify their learning profiles. Subjects and methods. The consent of the author was solicited to translate and apply the VARK questionnaire to 530 students and 27 professors of Public Health course, which is given at first year medical students. Fisher's exact test was used to identify the existence of differences between the learning profiles of the professors and the learning modalities of the students and then both compared with the averages of grades separated by group. By means of inferential analysis the association between gender and group characteristics with the average grades looked for. Results. $31 \%$, of the students displayed a single modality of learning, and $69 \%$ declared more than one learning modality; and of these last ones 14\% said they development all four modalities. It is interesting to note that $41 \%$ are visual/kinesthetic and $64 \%$ of the students displayed of one to two modalities. About the teachers $26 \%$ used only one teaching modality, $74 \%$ used more than one, and $4 \%$ used all four modalities. There were no statistical significance differences between women and men neither between, morning and afternoon groups nor with high performance groups. Conclusions. This first approach allowed us to have a general vision of the preferences of learning in the studied population. When the teacher identifies how their students learn and also recognizes his/her own, he/she can enrich their educational practice, and approach it to the necessities of the students and, that way improve the teaching process and better the student's results.

Key words. Learning strategies. Student. Teacher. VARK.

\section{Introducción}

Para apoyar a los alumnos en el desarrollo de habilidades que mejoren su aprendizaje, en las últimas tres décadas se ha generado una considerable producción de publicaciones en torno a
Facultad de Medicina. Universidad Nacional Autónoma de México. México DF, México.

\section{Correspondencia} Dra. María Eugenia Ponce de León Castañeda. Facultad de Medicina. Universidad Nacional Autónoma de México. Coordinación de Planes de Estudio, edif. B, piso 1. Ciudad Universitaria 3000 CP 04510. Col. Copilco, Delegación Coyoacán. México DF, México.

E-mail maeugenia.poncedeleón@ gmail.com. 
las habilidades, orientaciones y estrategias que son utilizadas por los estudiantes en el aprendizaje de la medicina.

Se ha visto que cada alumno aprende de manera distinta a los demás, utiliza diversas estrategias, aprende con diferentes velocidades e incluso con mayor o menor eficacia, aun con las mismas motivaciones, similar nivel de instrucción, la misma edad o estén estudiando el mismo tema [1].

Si bien la manera de aprender evoluciona y cambia constantemente, algunas características de la forma de apropiarse del conocimiento son relativamente estables y pueden ser susceptibles de identificarse y mejorarse [2].

Por otra parte, son varios los estudios que confirman la relación entre los estilos de aprendizaje y el éxito académico, como resultado de la respuesta de los alumnos a diferentes métodos de enseñanza. Algunos investigadores han encontrado evidencias de que presentar la información mediante distintos estilos lleva a una instrucción más efectiva [1].

$\mathrm{Al}$ respecto, Alonso, Gallego y Honey, después de analizar distintas investigaciones, llegaron a la conclusión de que parece suficientemente probado que los estudiantes aprenden con más efectividad cuando se les enseña con base a sus estilos de aprendizaje predominantes [3].

De la misma manera, Sarah Sharif-Paz menciona que los alumnos suelen recordar un $20 \%$ de lo que leen, un $30 \%$ de lo que oyen, un $40 \%$ de lo que ven, un $50 \%$ de lo que dicen y un $60 \%$ de lo que hacen, porcentaje que se incrementa a un $90 \%$ cuando dicen, oyen, ven y hacen [4].

Las modalidades o estilos de aprendizaje se definen como la manera en que aprendemos y nos adaptamos al ambiente [5]. Son las conductas que indican las preferencias de cómo aprendemos e integran el área cognitiva, afectiva, los rasgos de la personalidad y la orientación particular que tenemos al percibir, interpretar y responder ante la información.

Diversas aportaciones sirvieron de base para el desarrollo de los cuestionarios de identificación de los estilos de aprendizaje: Dewey (1938) señaló que los estudiantes aprenden mejor si se incluye un componente de experiencia en el proceso de aprendizaje; posteriormente, Lewin (1951) encontró que un entorno de aprendizaje activo desempeña un papel importante. Por otra parte, Piaget (1971) amplió la investigación concluyen- do que la inteligencia es un aspecto del dinamismo entre la persona y el entorno de aprendizaje.

Estos descubrimientos sirvieron de preparación para el desarrollo de numerosos cuestionarios sobre estilos de aprendizaje. Entre ellos cabe destacar el Learning Style Inventori (LSI) de Kolb, el LSI de Dunn y el Myers-Briggs Type Indicator [6]. De igual manera, pero con un enfoque más hacia lo cualitativo, están los trabajos realizados en el Reino Unido por Ramsden y Entwistle (1981), con sus orientaciones del aprendizaje (superficial, profundo y estratégico), estudiadas mediante el Approaches to Study Inventory (ASI) [7] y el Course Experience Questionnaire (CEQ). También se encuentran las aportaciones realizadas por Biggs con su Study Process Questionnaire (SPQ) [8], y la Theory Planned Behaviour, aportada por Ajzen y que se ha utilizado en la elaboración de numerosos cuestionarios [9].

Es importante reconocer el gran valor que representa para el docente identificar los estilos de aprendizaje de los estudiantes, con lo cual podrá diversificar sus estrategias de enseñanza y lograr con ello que sus alumnos aprendan de manera más efectiva y sencilla, lo que redundará en una gran satisfacción y motivación del profesor.

Neil Fleming [5], como inspector de enseñanza secundaria en Nueva Zelanda, en la década de los ochenta, se formuló dos preguntas: por qué algunos estudiantes aprenden a pesar de recibir una calidad de enseñanza muy pobre y por qué algunos estudiantes no aprenden a pesar de recibir una enseñanza de alta calidad. Sus preguntas empezaron a tener respuesta en los trabajos de Buzan, Kolb, Myers-Briggs y los neurolingüistas Bandler y Grinders, aportaciones con las cuales Fleming construye un cuestionario, VARK, que evalúa cuatro estilos de aprendizaje: visual, auditivo, lectoescritor y cinestésico.

El cuestionario VARK tiene como propósito identificar una de las dimensiones que forman parte del estilo de aprender. Proporciona un perfil de las preferencias de cómo se trabaja la información. El autor denomina 'preferencia modal' a la manera en que a las personas les gusta percibir la información y cómo ofrecen las respuestas.

Para percibir el mundo se utilizan los sentidos: vista, oído, tacto, olfato y gusto. En el aprendizaje académico lo más usual es ver, escuchar y hablar, $\mathrm{y}$ con menos frecuencia, gustar, tocar y oler. Ningún estudiante o profesor se restringe a una sola modalidad para recibir o enviar información; 
puede ocurrir que alguien, para aprender, utilice simultáneamente todos los sentidos, pero también se pueden presentar casos en que existan preferencias dominantes y algunas de ellas estén sin uso. También puede haber estudiantes y profesores con una sola preferencia muy marcada o fuerte y que las otras modalidades tengan una presencia muy débil [5].

El cuestionario VARK se integra con las cuatro categorías o preferencias mencionadas, además de sus combinaciones, las cuales se miden a través de 13 preguntas con cuatro opciones de respuesta. Es un cuestionario breve que facilita la concentración de quienes responden [10].

La preferencia visual se refiere a adquirir la información a través de imágenes, representaciones gráficas que incluyan cuadros, flechas o símbolos.

En la preferencia auditiva predomina la capacidad de 'escuchar' y a los estudiantes se les facilita aprender en conferencias y en el díalogo con sus compañeros.

En la preferencia de lectura/escritura se percibe más fácilmente la información mostrada en palabras escritas. Esta modalidad suele ser fuerte en muchos profesores y es característica de la enseñanza occidental.

En la preferencia cinestésica predomina el aprendizaje práctico, se facilita el aprender haciendo o al experimentar en el mundo. En esta modalidad se utilizan simultáneamente varios sentidos y se caracteriza por un fuerte contacto con la realidad.

Fleming, al aplicar el cuestionario, encontró diferencias entre los estudiantes y los profesores. Los profesores tienden a la preferencia de lectura/ escritura, y los estudiantes, a la cinestésica. Asimismo, la mayoría de los estudiantes (alrededor del $70 \%$ ) son multimodales, ya sea en combinación de dos, tres o las cuatro preferencias. Sin embargo, Fleming afirma que estos resultados se pueden modificar según la disciplina que se esté estudiando; así, observó que los estudiantes del área de humanidades son preferentemente del estilo lectura/escritura, y los del área de farmacología, multimodales. El autor esperaba que también existieran diferencias en las diversas culturas.

Una vez obtenidos los resultados del cuestionario, se ofrecen sugerencias para aprovechar al máximo las preferencias identificadas. Lo importante es conocer las propias preferencias, hacer una reflexión que permita optimizar la manera en que se recibe la información y favorecer que los profesores, en su forma de enseñar, ofrezcan mejores respuestas a la diversidad de modalidades en sus estudiantes.

En la Facultad de Medicina de la Universidad Nacional Autónoma de México (UNAM), los alumnos que no aprueban su pase al año siguiente y las deserciones en el primer año son elevados. Una de las causas de esta situación es que los estudiantes no cuentan con las estrategias de aprendizaje adecuadas para hacer frente a la gran cantidad y complejidad de información que demanda la formación del médico [11].

Para apoyar a los estudiantes con estrategias de aprendizaje adecuadas es necesario, primero, proporcionarles elementos para que puedan reconocer su propia manera de aprender, y de esta forma tener posibilidades de aprovechar el propio potencial, mejorarlo y enriquecerlo.

La carrera de médico cirujano está configurada por 24 asignaturas obligatorias con 433 créditos, más 16 créditos en asignaturas de libre elección. La Facultad de Medicina, en el año del estudio, atendió a un total de 6.340 alumnos, 20 de los cuales eran extranjeros, 4.058 (64\%) eran mujeres y 2.282 (36\%) eran varones, con un promedio de edad de 22 años.

Salud Pública I es una asignatura obligatoria que se imparte en el primer año de carrera. Tiene una matrícula de 1.197 alumnos, 51 profesores y 38 grupos (29 matutinos y 9 vespertinos). La asignatura está integrada por tres unidades temáticas: fundamentos de la salud pública, alimentación y nutrición humana, y metodología de la investigación. Su principal propósito es ofrecer al estudiante la oportunidad de discutir los fundamentos teóricos del proceso salud-enfermedad y se orienta, en el marco del método científico, al conocimiento de los problemas colectivos de salud y a la conducción de actividades para solucionarlos.

La asignatura tiene uno de los índices más bajos de alumnos que no pasan su pase al año siguiente en el primer año de carrera y la opinión general de los estudiantes es que sus contenidos no son de alta complejidad. Las estrategias de enseñanza y aprendizaje que se sugieren a los profesores para ser aplicadas cuando lo consideren conveniente son: metodología centrada en la solución de problemas, vinculación teóricopráctica de los conocimientos, búsqueda y análisis crítico de información en fuentes originales y bancos automatizados, aplicación de técnicas de 
enseñanza que favorezcan la participación de los alumnos y formación de grupos de alumnos para trabajar en equipo.

Los contenidos de la asignatura y las actividades recomendadas permiten suponer que si los estudiantes trabajan en pequeños grupos, realizan búsquedas de información y aplican los conocimientos adquiridos para solucionar problemas, entonces, de acuerdo con la propuesta de Fleming, los estudiantes cinestésicos, o quienes tienen una combinación que contenga el estilo cinéstesico, experimentarán este tipo de actividades como más interesantes y atractivas, lo cual puede influir en su aprovechamiento académico. En oposición, los estudiantes con un predominio del estilo auditivo, que prefieren adquirir la información a través de su capacidad de escucha, pueden sentirse menos motivados en este tipo de cursos.

Los propósitos de este estudio fueron identificar los perfiles de aprendizaje de los alumnos y de los profesores en la asignatura de Salud Pública y definir si existen semejanzas o diferencias entre las preferencias del profesor y las de los alumnos. Lo anterior se toma como hipótesis exploratoria debido a que es el primer acercamiento al fenómeno en esta facultad y en la carrera de médico cirujano en nuestro medio.

\section{Sujetos y métodos}

Se consiguió el consentimiento del autor para aplicar la versión traducida del cuestionario VARK a alumnos y profesores de 24 grupos (63\%) en la asignatura de Salud Pública correspondiente al primer año de la carrera de Medicina, en el período escolar 2005-2006 de la UNAM. $\mathrm{El}$ instrumento fue contestado por 530 alumnos (44\%) y 27 profesores (53\%).

La participación fue voluntaria. Los profesores correspondían a 16 grupos del turno matutino y 8 del vespertino, de un total de 36 .

Las respuestas al cuestionario se realizaron en hojas para lectura óptica, se procesaron de acuerdo con la metodología indicada por Fleming y se analizaron con un paquete estadístico comercial.

Mediante la puntuación en el cuestionario, los participantes se ubicaron, de acuerdo con su o sus perfiles, en algunas de las cuatro posibilidades exploradas (visual, auditivo, lectoescritor y cinestésico) y sus posibles combinaciones.
Las modalidades para cada uno de los grupos se asignaron cuando un $50 \%$ o más de los alumnos del grupo presentó la modalidad.

Se obtuvieron las calificaciones finales promedio por grupo en la asignatura y éstas se dividieron en dos categorías: baja $(\leq 7,9)$ y alta $(\geq 8,0)$.

Se utilizó la prueba exacta de Fisher para identificar la existencia de diferencias entre los perfiles de aprendizaje de los profesores con las modalidades de aprendizaje en los alumnos, y ambos, con los promedios de las calificaciones por grupo.

De la misma manera, mediante análisis inferencial, se buscó la asociación entre el género y las características de los grupos con el promedio de calificaciones.

\section{Resultados}

Se analizaron 530 cuestionarios de alumnos, 381 (72\%) de grupos matutinos y 149 (28\%) de vespertinos; 83 (16\%) correspondieron a grupos de alto rendimiento. 323 (61\%) fueron contestados por mujeres y 207 (39\%) por hombres; las edades fluctuaron entre 17 y 29 años.

De los 27 profesores participantes, 16 (67\%) fueron de los grupos matutinos y 8 (33\%) de los vespertinos; tres grupos tuvieron dos profesores durante el curso y 3 (11\%) correspondieron a grupos de alto rendimiento.

Los alumnos que presentaron una sola modalidad de aprendizaje fueron 169 (31\%); 361 (69\%) manifestaron más de una; de estos últimos, 74 (14\%) presentaron el desarrollo de las cuatro modalidades. Destaca que 218 alumnos (41\%) son visual/cinestésicos. 341 (64\%) alumnos presentaron de una a dos modalidades (Tabla I).

Siete profesores (26\%) tuvieron una sola modalidad de aprendizaje y los 20 restantes (74\%) presentaron más de una; de estos últimos, uno (4\%) aplica las cuatro modalidades. Destaca que 10 profesores $(37 \%)$, de la misma manera que los alumnos, también son visual/cinestésicos. 17 (63\%) profesores presentaron de una a dos modalidades (Tabla II, Figura).

Cincuenta y dos alumnos (10\%) presentaron modalidades iguales a las del profesor, 145 (27\%) abarcaron las modalidades del profesor y además tuvieron alguna otra más, 254 (48\%) manifestaron algún rasgo presentado por el docente y 79 
Tabla I. Perfil de preferencias en la forma en que los alumnos asimilan el conocimiento.

\begin{tabular}{|c|c|c|c|c|c|c|c|}
\hline 1 modalidad & $n$ & 2 modalidades & $n$ & 3 modalidade & $n$ & 4 modalidades & $n$ \\
\hline v & 55 & RV & 22 & RVK & 38 & VARK & 74 \\
\hline A & 14 & VK & 84 & RVA & 15 & & \\
\hline $\mathrm{R}$ & 21 & KA & 28 & RKA & 19 & & \\
\hline \multirow[t]{3}{*}{ K } & 79 & RK & 19 & VKA & 43 & & \\
\hline & & VA & 13 & & & & \\
\hline & & RA & 6 & & & & \\
\hline \multicolumn{2}{|c|}{$169(31 \%)$} & \multicolumn{2}{|c|}{$172(33 \%)$} & \multicolumn{2}{|c|}{$115(22 \%)$} & \multicolumn{2}{|c|}{$74(14 \%)$} \\
\hline
\end{tabular}

(15\%) fueron totalmente distintos en sus modalidades a las del profesor (Tabla III).

En cuanto a las comparaciones de las variables estudiadas en relación con el promedio grupal, no se encontraron diferencias estadísticamente significativas cuando los alumnos con la modalidad idéntica a la del profesor se compararon con los no idénticos. De la misma manera, no se presentaron diferencias cuando los alumnos con modalidades que incluían a las de los profesores, y que además presentaron alguna otra más, se compararon con los otros alumnos.

Tampoco se encontraron diferencias estadísticamente significativas cuando se comparó a los alumnos que presentaron algún rasgo en su modalidad, el cual también se presentaba en el docente. Lo mismo sucedió cuando se comparó el grupo de alumnos con modalidades distintas a las del profesor con los demás alumnos $(p=0,139$, $p=0,444, p=0,662$ y $p=1,0$, respectivamente).

No se encontraron diferencias estadísticamente significativas entre las mujeres y los hombres ni entre los grupos matutinos, vespertinos o de alto rendimiento ( $p=0,092$ y $p=0,173$, respectivamente).

Tres grupos mostraron sólo una modalidad, la misma que se manifestó en el 50\% o más de los alumnos, y ésta fue la cinestésica, mientras que las modalidades de aprendizaje de los profesores para cada uno de estos grupos fueron: cinestésica- lectoescritura, lectoescritura-visual y lectoescritura. Cada uno de estos grupos se ubicó en una de las tres categorías de los promedios.

En sólo dos grupos, las cuatro modalidades se manifestaron en más del 50\% de los alumnos, en tanto que de los profesores de estos grupos, uno tenía desarrolladas las cuatro modalidades de aprendizaje, pero el otro sólo utilizaba una modalidad. Los promedios de ambos grupos se ubicaron en el estrato bajo.

\section{Discusión}

Este primer acercamiento exploratorio permitió tener una visión general de las preferencias de aprendizaje de la población estudiada. El patrón de las modalidades de aprendizaje encontradas en los alumnos principalmente fue cinestésico, visual y lectoescritura. También el perfil de aprendizaje de los profesores principalmente fue visual-cinestésico y lectoescritura. La mayor parte de los alumnos y de los profesores utiliza estrategias de aprendizaje multimodales, lo cual concuerda con lo encontrado por Fleming [5]. Hay un grupo importante de alumnos y profesores que utilizan una sola estrategia o máximo dos, y muy pocos que han desarrollado las cuatro modalidades. 


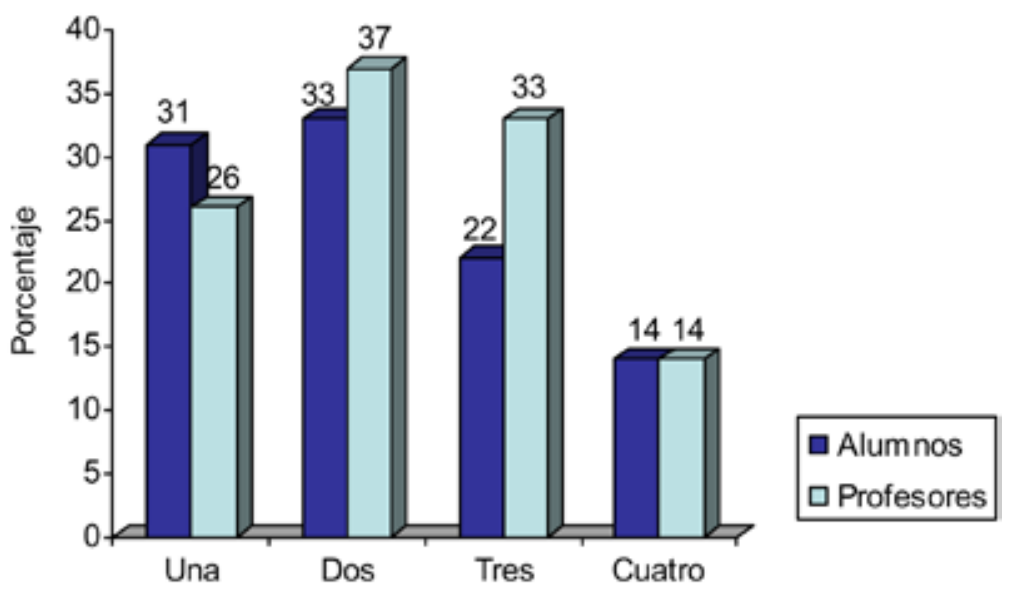

Figura. Porcentaje de alumnos y profesores por número de preferencias.

Tabla II. Perfil de preferencias en la forma en que los profesores asimilan el conocimiento.

\begin{tabular}{|c|c|c|c|c|c|c|c|}
\hline 1 modalidad & $n$ & 2 modalidades & $n$ & 3 modalidades & $n$ & 4 modalidades & $n$ \\
\hline V & 3 & RV & 3 & RVK & 4 & VARK & 1 \\
\hline A & 0 & VK & 5 & RVA & 0 & & \\
\hline $\mathrm{R}$ & 2 & KA & 0 & RKA & 2 & & \\
\hline \multirow[t]{3}{*}{ K } & 2 & RK & 1 & VKA & 3 & & \\
\hline & & VA & 1 & & & & \\
\hline & & RA & 0 & & & & \\
\hline $26 \%$ & & $37 \%$ & & $33 \%$ & & $4 \%$ & \\
\hline
\end{tabular}

No se encontraron diferencias estadísticamente significativas en cuanto al promedio del grupo y el apego o no de las modalidades de los alumnos con las de los profesores, ni en cuanto a género o características del grupo. Sin embargo, la tendencia de las pruebas estadísticas puede apoyar la hipótesis de que, en la medida en que los alumnos presentan una modalidad idéntica a la del profesor, puede manifestarse un mayor promedio de calificaciones grupal, lo cual seguramente conse- guiría comprobarse incrementando el número de participantes en el estudio (Tabla III).

Para este estudio se utilizó la versión en español del cuestionario elaborada por el Dr. Óscar Soria, con la autorización del autor, por lo que no se consideró pertinente nuevamente su validación. Asimismo, no fue posible obtener la fiabilidad de los resultados.

Los resultados encontrados en este acercamiento exploratorio proporcionan elementos para rea- 
Tabla III. Valores estadísticos de las modalidades de alumnos y profesores en relación con el promedio de calificaciones en la asignatura.

\begin{tabular}{lcc}
\hline Modalidad del grupo & N. ${ }^{\circ}$ de alumnos & Prueba exacta de Fisher \\
\hline Igual a la del profesor & 52 & 0,139 \\
\hline Incluye la del profesor y además alguna otra & 145 & 0,444 \\
\hline Con algún rasgo que presenta también el profesor & 254 & 0,662 \\
\hline Totalmente distinta a la del profesor & 79 & 1,0 \\
\hline
\end{tabular}

lizar un estudio con una mayor población y con mejor control de las variables, para poder destacar la importancia de identificar los estilos de aprendizaje de los alumnos y su adecuación con las estrategias de enseñanza, así como su repercusión en el aprovechamiento escolar. Cuando el docente identifica cómo aprenden sus alumnos y también reconoce su propia manera de enseñar y aprender, puede enriquecer su práctica docente, acercarla a las necesidades de sus alumnos y, con ello, mejorar el proceso de enseñanza y los resultados del aprendizaje.

\section{Bibliografía}

1. Revilla D. Estilos de aprendizaje. Temas de Educación, Segundo Seminario Virtual 1998. URL: http://www. pucp.edu.pe/ temas/estilos.html.

2. Saarikoski L, Salojärvi S, Del Corso D. The DE: an environment for the development of learner-oriented customised educational packages. International Conference on Information Technology Based Higher Education and Training. Kumamoto, Japan, July 2001. URL: http// www.eecs.kumamoto-u.ac.jp/ithet01/proceedings.htm.

3. Gallego R, Martínez C. Estilos de aprendizaje y e-learning. Hacia un mayor rendimiento académico. URL: www. um.es/ead/red/7.

4. Quirós G. La literatura en la clase de idiomas. Revista de Artes y Letras 2005; 29: 229-306.

5. Fleming ND. Teaching and learning styles: VARK strategies. Christchurch: N.D. Fleming; 2001.

6. Matthews BD, Hamby VJ. A comparison of the learning styles of high school and college/university students. The Clearing House 1995; 68: 257-61.

7. Richardson J. Approaches to striding and perceptions of academic quality in a short web based course Br J Educ Technol 2003; 34: 433-42.

8. Biggs J. The Study Process Questionnaire (SPQ): manual. Hawthorn: Australian Council for Educational Research; 1987.

9. Francis JJ, Eccles MP, Johnston M, Walker AE, Grimshaw JM, Foy R, et al. Constructing questionnaires based on the theory of planned behaviour. A manual for health services researchers. Newcastle upon Tyne: Centre for Health Services Research, University of Newcastle; 2004.

10. Fleming ND, Mills C. Not another inventory, rather a catalyst for reflection. To Improve the Academy 1992; 11: 137-55.

11. Varela M, Fortoul T. El reto de los estudiantes de medicina. 1 ed. México: Médica Panamericana/UNAM; 2003. 